\title{
Methadone and Morphine during Anesthesia Induction for Cardiac Surgery. Repercussion in Postoperative Analgesia and Prevalence of Nausea and Vomiting
}

\author{
Artur Udelsmann, TSA ${ }^{1}$, Fernanda Gardini Maciel ${ }^{2}$, Derli Conceição Munhoz Servian ${ }^{3}$, Eder Reis, TSA ${ }^{4}$, \\ Teresinha Maria de Azevedo, TSA ${ }^{4}$, Marcos De Simone Melo ${ }^{5}$
}

\begin{abstract}
Summary: Udelsmann A, Maciel FG, Servian DCM, Reis E, Azevedo TM, Melo MS - Methadone and Morphine during Anesthesia Induction for Cardiac Surgery. Repercussion in Postoperative Analgesia and Prevalence of Nausea and Vomiting.

Background and objectives: Pain is an aggravating factor in postoperative morbidity and mortality especially in large size surgeries. Methods to effectively fend pain collide with elevated costs and for this reason they are not accessible in every service. The option would be the use of an opioid with long half-life, such as methadone. The objective of the present study was to compare the requirements of postoperative analgesia in patients who received methadone, morphine, or placebo during anesthetic induction, besides the prevalence of postoperative nausea and vomiting.
\end{abstract}

Methods: Fifty-five patients scheduled for cardiac surgery were divided into three groups and they received during anesthetic induction 20 mg of methadone, $20 \mathrm{mg}$ of morphine, or placebo. At the end of surgery, patients were transferred to the ICU where the following parameters were evaluated: duration of anesthesia, time until extubation, time until the need of the first analgesic, number of doses required in 24 hours, assessment of analgesia by the patient, and prevalence of nausea/vomiting.

Results: Differences in the duration of anesthesia and time until extubation were not observed. The first dose of analgesic in patients who received methadone was administered later than in patients in the other two groups. The need of analgesics in the methadone group was lower, quality of analgesia was better, and prevalence of nausea and vomiting was also lower.

Conclusions: Methadone during anesthetic induction was effective for analgesia in large size surgeries. Lower incidence of nausea and vomiting was observed in the methadone group and therefore it is a low cost option available among us that should be stimulated.

Keywords: Methadone; Morphine; Pain, Postoperative; Thoracic Surgery; Postoperative Nausea and Vomiting.

\section{INTRODUCTION}

Frequently one reads that postoperative analgesia is inadequate. Intramuscular injection of opioids with elevated clearance and relatively short half-life, such as morphine, produce important fluctuations in opioid serum levels with values oscillating from inadequate analgesia to toxic levels. In this case, the alternative would be to administer intravenous infusion of

Received from CET/Anesthesiology Department of the Faculdade de Ciências Médicas (FCM) of Universidade de Campinas (Unicamp), Brazil.

1. Associate Professor; Anesthesiology Department of the Faculdade de Ciências Médicas (FCM) of Universidade de Campinas (Unicamp)

2. R3; Unicamp

3. Professor; Physician, Anesthesiology Sector of the Hospital das Clínicas da Unicamp

4. Physician, Anesthesiology Sector of the Hospital das Clínicas da Unicamp

5. PhD Student in Surgical Sciences at FCM/Unicamp; Physician of the Anesthesiology Sec-

tor of Hospital das Clínicas da Unicamp

Submitted on February 27, 2011.

Approved on April 4, 2011.

Correspondence to:

Dr. Artur Udelsmann

Av. Prof. Atílio Martini, 213

13083830 - Campinas, SP, Brazil

E-mail:audelsmann@yahoo.com.br analgesics, either by demand or continuous. Both methods, however, require a costly apparatus. An alternative method that promotes continuous analgesia without the problems associated with infusion techniques would be a long half-life agent with low clearance: methadone. Methadone is a synthetic opioid with long latency and duration used for several years to treat drug addiction ${ }^{1}$ and "rediscovered" as analgesic in the treatment of chronic pain ${ }^{2}$, cancer pain ${ }^{3}$, and also for postoperative analgesia both in adults ${ }^{4}$ and children ${ }^{5}$, including among anesthesiologists ${ }^{6}$. Long-lasting relief was observed, since its plasma concentration decreases slowly as a consequence of its slow metabolism.

Methadone is a $m u$ receptor antagonist, exerting simultaneously an antagonism of the activity of NMDA (N-methyl$\mathrm{d}$-aspartate) receptors and inhibiting the reuptake of catecholamines ${ }^{7}$. It has a long half-life, but a large individual variation is observed 8 . Parenterally, effects are observed within 10 to 20 minutes, lasting 4 to 8 hours ${ }^{9}$, or up to 26 hours ${ }^{4}$. A mean of $90 \%$ is bound to plasma proteins, and it has hepatic metabolism into inactive metabolites. Its parenteral potency is equivalent to that of morphine ${ }^{8}$, and it has a halflife of approximately 22 hours, ranging from 13 to 50 hours, with great individual variation ${ }^{1}$. Due to its long duration, it has become an interesting drug in the treatment of postoperative 
pain, especially in large size surgeries with prolonged recovery time, such as cardiac surgeries. In adults the recommended dose is $20 \mathrm{mg}$ at the time of anesthesia induction ${ }^{4,9,10}$, while in children ${ }^{5,7}$, it is 0.2 to $0.3 \mathrm{mg} \cdot \mathrm{kg}^{-1}$. Although used in other countries only recently it has become available for parenteral use among us. The objective of the present study was to evaluate analgesia, in the first 24 postoperative hours of cardiac surgeries, by the same dose of methadone and morphine administered at the onset of anesthesia, as well as the incidence of nausea and vomiting, comparing these methods with the lack of administration of analgesic during anesthetic induction, which is the current practice in our institution.

\section{METHODS}

After approval by the Ethics on Research Committee and signing of the informed consent, 55 patients of both genders, ages 14 to 80 years, ASA III or IV, undergoing cardiac surgery with extracorporeal circulation, participated in this study. The sample size was based on prior pilot study. Patients who used illicit drugs or antidepressants, those with associated psychiatric diseases, with history of allergies to any of the drugs, and those who had to remain intubated during the postoperative period for more than 24 hours were excluded. Patients were randomly and double-blindly divided into three groups: ME (methadone), MO (morphine), and CTL (control). In the operating room all were monitored with invasive blood pressure, cardioscope, pulse oximetry, capnography, and central venous pressure. Anesthetic induction was performed with sufentanil $0.1 \mu \mathrm{g} \cdot \mathrm{kg}^{-1}$, midazolam $0.1 \mathrm{mg} \cdot \mathrm{kg}^{-1}$, and pancuronium $0.1 \mathrm{mg}^{\mathrm{kg}}{ }^{-1}$.
Soon after induction, and without the knowledge of the anesthesiologist and the patient, $20 \mathrm{mg}$ of morphine (MO), $20 \mathrm{mg}$ of methadone (ME), or $2 \mathrm{~mL}$ of saline (CTL) were administered. Anesthesia was maintained with sufentanil $0.01 \mu \mathrm{g} \cdot \mathrm{kg}^{-1} \cdot \mathrm{min}, 0.5 \%$ of isoflurane, and $0.03 \mathrm{mg} \cdot \mathrm{kg}^{-1}$ of pancuronium whenever it was clinically necessary. At the end of surgery, the patient was transferred to the ICU, intubated. On this unit, professionals did not know in which group the patient was included and, according to criteria of the medical and nursing team of this unit, after extubation $0.03 \mathrm{mg} \cdot \mathrm{kg}^{-1}$ of IV morphine was administered whenever necessary. At the end of this period the patient was asked to evaluate the quality of analgesia through the visual analogue scale.

Duration of anesthesia, time until extubation, time until the administration of the first dose of analgesic, the number of doses of analgesic required during this period, and the prevalence of nausea and vomiting were recorded. The SPSS for Windows, version 13.0, was used for the statistical analysis. A descriptive analysis of the quantitative and qualitative variables was performed; to determine the association among the different qualitative variables Fisher's exact test or the Chi-square test was used. Quantitative variables were compared among groups using the non-parametric Kruskal-Wallis test and Tukey test. A level of significance of $5 \%$ was adopted.

\section{RESULTS}

All three groups were homogenous regarding anthropometric data, physical status ASA, and gender distribution (Table I).

Differences on time $(T)$ of anesthesia and until extubation were not observed among the three groups. The first dose of analgesic in the ME group was required after longer time $(p=0.0261)$ (Table II).

Table I - Anthropometric Data, Physical Status ASA, and Gender Distribution

\begin{tabular}{llllll}
\hline Group & $\#$ & Age & BMI & ASA III / IV & Gender M / F \\
\hline ME & 18 & $57.89 \pm 14.6$ & $27.4 \pm 3.9$ & $9 / 9$ & $11 / 7$ \\
MO & 19 & $56.63 \pm 13.9$ & $25.67 \pm 3.2$ & $9 / 10$ & $11 / 8$ \\
CTL & 18 & $56.83 \pm 10.6$ & $27.4 \pm 5.8$ & $7 / 11$ & $8 / 10$ \\
\hline
\end{tabular}

ME: Methadone; MO: Morphine; CTL: Control.

Table II - Time (T) of Anesthesia, T of Extubation, $T$ for $1^{\text {st }}$ Dose of Analgesic in Minutes

\begin{tabular}{llcc}
\hline Group & T anesthesia & T extubation & T 1 st dose of analgesic \\
\hline ME & $285.28 \pm 50.13$ & $430.83 \pm 304.35$ & $855.17 \pm 593.39 *$ \\
MO & $286.05 \pm 57.6$ & $358.95 \pm 179.15$ & $625.53 \pm 525.23$ \\
CTL & $303.11 \pm 70.12$ & $341.67 \pm 144.41$ & $507.56 \pm 413.04$ \\
\hline
\end{tabular}

ME: Methadone; MO: Morphine; CTL: Control. 
Table III - Number of Patients Who Required Analgesics and Number of Doses

\begin{tabular}{lllll}
\hline Group & $\#$ & $\begin{array}{l}\text { Patients requiring } \\
\text { analgesics }\end{array}$ & Number of doses & VAS \\
\hline ME & 18 & $10^{*}$ & $0.89 \pm 1.02$ & $0.5 \pm 0.71^{*}$ \\
MO & 19 & 14 & $1.32 \pm 1.05$ & $1.84 \pm 1.38$ \\
CTL & 18 & 17 & $2.39 \pm 0.85^{*}$ & $2.83 \pm 2.18$ \\
\hline
\end{tabular}

ME: Methadone; MO: Morphine; CTL: Control.

Table IV - Incidence of Nausea and/or Vomiting (PONV)

\begin{tabular}{lll}
\hline Group & $\#$ & patients with PONV \\
\hline ME & 18 & $1^{*}$ \\
MO & 19 & 6 \\
CTL & 18 & 9 \\
\hline
\end{tabular}

ME: Methadone; MO: Morphine; CTL: Control.

The number of patients who needed analgesics was significantly lower in the ME group ( $p=0.025)$; the number of doses of analgesic in 24 hours in the CTL group was significantly greater than in the other two groups $(p<0.001)$; the quality of analgesia after 24 hours according to the patient was significantly better in the ME group $(p<0.01)$ (Table III).

In the ME group the incidence of nausea and/or vomiting was significantly lower than in the other two groups $(p=0.013)$ (Table IV).

\section{DISCUSSION}

This study showed the interest in using methadone in postoperative analgesia in cardiac surgeries. Its use during anesthetic induction allowed more prolonged analgesia; the first dose of analgesic in patients who received methadone was administered almost 4 hours after the first dose in the morphine group and 6 hours after the control group. An increase in the time until extubation was not observed.

In the first 24 postoperative hours the number of patients who required analgesia was significantly lower in the methadone group, as well as the assessment of the quality of analgesia by the patient, which translated in this group a significantly lower VAS score. The number of doses of anal- gesic required was greater in the group that did not receive methadone or morphine during anesthetic induction; in this aspect, although those who received methadone had less analgesic administered than those who received morphine, this difference was not significant, requiring a greater sample size to demonstrate it.

The lower requirement of postoperative opioids in patients who received methadone might explain the lower incidence of nausea and vomiting in this group, which is interesting in interventions with greater algesic potential, such as sternotomies.

Our results opposed those of Gottschalk et al. ${ }^{11}$, who observed lower postoperative opioid consumption only 48 hours after the administration of methadone. These authors suggested that the main advantage of methadone would be in reality its capacity to attenuate the tolerance to opioids administered and hyperalgesia due to its antagonistic action in NMDA receptors, since, although the mechanisms of tolerance are yet to be determined, they seem to involve their activation ${ }^{12}$.

Methadone is metabolized by type I cytochrome P450 enzymes and their products are inactive and eliminated on the urine and feces ${ }^{13}$; it does not accumulate in patients with kidney failure, and therefore does not require dose reduction, but at the same time it is eliminated in relatively small quantities by hemodialysis ${ }^{2,14}$. Its most important adverse effects include the possibility of increase in the QT interval on the electrocardiogram, which can lead to Torsades de Pointes ventricular arrhythmias 8,13,14 in case of large doses; and erythema and dermal hardening when used subcutaneously ${ }^{13,15}$.

This study highlights the interest in a little known drug by anesthesiologists, though available for many years, since synthesized in Germany in 1937. It is known to be effective but not very used for postoperative analgesia, although it is an interesting and low cost alternative when compared to other methods used in large size surgeries. 